\title{
Asiatic acid attenuates cardiac hypertrophy by blocking transforming growth factor- $\beta 1$-mediated hypertrophic signaling in vitro and in vivo
}

\author{
LINJIE SI ${ }^{1,2 *}$, JING XU $^{1,2^{*}}$, CHENLONG YI ${ }^{1,2}$, XIAOHAN XU ${ }^{1,2}$, FANG WANG $^{3}$, \\ WEIJUAN GU ${ }^{3}$, YUQING ZHANG $^{4}$ and XIAOWEI WANG ${ }^{1,2}$
}

\author{
${ }^{1}$ Department of Thoracic and Cardiovascular Surgery, The First Affiliated Hospital of Nanjing Medical University, \\ ${ }^{2}$ Institute of Integrated Medicine, Nanjing Medical University, ${ }^{3}$ Department of Cardiology, The First Affiliated Hospital \\ of Nanjing Medical University, Nanjing, Jiangsu 210029; ${ }^{4}$ Department of Cardiology, The Affiliated Jiangning Hospital \\ of Nanjing Medical University, Nanjing, Jiangsu 211100, P.R. China
}

Received January 27, 2014; Accepted April 30, 2014

DOI: $10.3892 /$ ijmm.2014.1781

\begin{abstract}
Cardiac hypertrophy is a major cause of morbidity and mortality worldwide. Transforming growth factor- $\beta 1$ (TGF- $\beta 1$ ) signaling has been considered as a trigger causally contributing to pathological cardiac hypertrophy. Asiatic acid (AA) is a triterpenoid compound extracted from Centella asiatica and exhibits a variety of pharmacological effects. In this study, we investigated the anti-hypertrophic effects and mechanisms of action of AA in a TGF- $\beta 1$-stimulated hypertrophic response using cultured neonatal cardiomyocytes in vitro and in a mouse model of cardiac hypertrophy induced by pressure overload in vivo. Treatment with AA markedly attenuated the TGF- $\beta 1$-induced hypertrophic responses of cardiomyocytes as reflected by reduction in the cardiomyocyte surface area and the inhibition of atrial natriuretic peptide (ANP) mRNA expression. The protective effects of AA on hypertrophic cardiomyocytes were associated with the blocking of p38 and extracellular signal-regulated kinase (ERK)1/2 phosphorylation and the reduction of nuclear factor $-\kappa \mathrm{B}(\mathrm{NF}-\kappa \mathrm{B})$ binding activity. In vivo experiments indicated that the administration of AA prevented
\end{abstract}

Correspondence to: Professor Xiaowei Wang, Department of Thoracic and Cardiovascular Surgery, The First Affiliated Hospital of Nanjing Medical University, 300 Guangzhou Road, Nanjing, Jiangsu 210029, P.R. China

E-mail: wangxiaowei@njmu.edu.cn

Professor Yuqing Zhang, Department of Cardiology, The Affiliated Jiangning Hospital of Nanjing Medical University, 168 Gushan Road, Nanjing, Jiangsu 211100, P.R. China

E-mail: zyq-zhq@163.com

${ }^{*}$ Contributed equally

Key words: asiatic acid, cardiac hypertrophy, transforming growth factor- $\beta 1$, mitogen-activated protein kinase, nuclear factor- $\kappa B$ cardiac hypertrophy and dysfunction induced by pressure overload. It was found that AA markedly reduced the excessive production of TGF- $\beta 1$ in the hypertrophic myocardium, blocked the phosphorylation of p38 and ERK1/2 and inhibited the activation of $\mathrm{NF}-\kappa \mathrm{B}$. Our data suggest that AA may be a novel therapeutic agent for cardiac hypertrophy. The inhibition of TGF- $\beta 1$-mediated hypertrophic signaling may be the mechanism through which AA prevents cardiac hypertrophy.

\section{Introduction}

Cardiac hypertrophy is characterized by the augmentation of the ventricular mass against pressure overload in disease settings, such as hypertension and aortic stenosis $(1,2)$. In the case of cardiac hypertrophy, although increased ventricular mass is initially a compensatory mechanism, sustained hypertrophy can ultimately lead to a decline in left ventricular function and heart failure. In this regard, cardiac hypertrophy has been considered as an independent risk factor for heart failure or sudden death $(2,3)$. Previous studies have explored the mechanisms involved in the development of pathological hypertrophy on a molecular and cellular level (1-3). This has led drug discovery research to the developement of effective therapies for pathological hypertrophy. However, current therapeutic agents are limited to halting the progression of this disease. Therefore, novel therapeutic strategies are required to inhibit the development of cardiac hypertrophy before heart failure develops.

In response to pressure overload, cardiomyocytes are subjected to mechanical stretching and release humoral factors through autocrine and paracrine signaling, such as through transforming growth factor- $\beta 1$ (TGF- $\beta 1$ ). TGF- $\beta 1$ is a pleiotropic and multifunctional cytokine (4) that serves as a master switch for cardiac hypertrophy. The activation of TGF- $\beta 1$-mediated hypertrophic signaling plays a crucial role in this process (5). The mitogen-activated protein kinase (MAPK) superfamily includes p38, extracellular signal-regulated kinase (ERK)1/2 (also known as p44/42) and c-Jun N-terminal kinase (JNK), all of which have been implicated as down- 
stream signaling targets of TGF- $\beta 1$ and causally contribute to cardiac hypertrophy $(4,6,7)$. Nuclear factor- $\kappa \mathrm{B}(\mathrm{NF}-\kappa \mathrm{B})$ is a well-known pluripotent transcription factor, which can be activated by MAPK signaling (8). Once activated, NF- $\kappa B$ stimulates gene expression and the products of inflammatory cytokines, and ultimately leads to cardiac hypertrophy $(8,9)$. It is evident that blocking NF- $\mathrm{KB}$ binding activity significantly attenuates cardiac hypertrophy (8). Therefore, the targeting of TGF- $\beta 1$-mediated hypertrophic signaling may constitute a suitable therapeutic intervention for pathological hypertrophy.

Asiatic acid (AA) is a pentacyclic triterpenoid that has been reported to exhibit a variety of pharmacological effects, including antioxidant (10), anti-inflammatory (11) and anti-apoptotic effects (12). Of note, a recent study demonstrated that AA inhibited liver fibrosis in vitro and in vivo, and the anti-hepatofibrotic effects of AA involved the blocking of the TGF- $\beta 1 /$ Smad signaling by reducing the TGF- $\beta 1$ expression levels (13). However, to the best of our knowledge, there is no study available to date on the effects of AA on cardiac hypertrophy. Thus, we hypothesized that AA may attenuate cardiac hypertrophy by blocking TGF- $\beta 1$-mediated hypertrophic signaling. To examine this hypothesis, in the present study, we investigated the anti-hypertrophic effects and mechanisms of action of AA using a TGF- $\beta 1$-stimulated hypertrophic cardiomyocyte model in vitro and a pressure overload-induced cardiac hypertrophy model in vivo.

\section{Materials and methods}

Materials. The purified natural product of AA (97\%) and dimethyl sulfoxide (DMSO), were obtained from SigmaAldrich (St. Louis, MO, USA). Recombinant human TGF- $\beta 1$ was obtained from PeproTech (Rocky Hill, NJ, USA). Dulbecco's modified Eagleæs medium (DMEM) and fetal bovine serum (FBS) were obtained from Gibco BRL Life Technologies, Inc. (Carlsbad, CA, USA). The cell counting kit-8 (CCK-8) assay kit was obtained from Dojindo Laboratories (Kumamoto, Japan). Primary antibodies against total and phosphorylated (p)-p38, ERK1/2 and JNK1/2 were obtained from Cell Signaling Technology (Berverly, MA, USA). The electrophoretic mobility shift assay (EMSA) kit was obtained from Pierce Biotechnology, Inc. (Rockford, IL, USA). Cytoplasmic and nuclear protein extraction kits were obtained from KeyGen Biotech Co., Ltd. (Nanjing, China). Unless otherwise indicated, all other chemicals and materials were obtained from Sigma-Aldrich.

Ethics statements. Animal handling and use complied with the Guide for the Care and Use of Laboratory Animals published by the US National Institutes of Health (NIH Publication no. 85-23, revised 1996) and were approved by the Animal Care and Use Committee of Nanjing Medical University (Nanjing, China). They were housed in a room maintained at $22^{\circ} \mathrm{C}$ with a 12:12 $\mathrm{h}$ light/dark cycle and provided with standard food and water ad libitum. The experiments were designed to minimize the pain inflicted and the number of animals used.

Primary cultures of neonatal rat ventricular myocytes. Hearts were immediately removed from 1- to 2-day-old neonatal Sprague-Dawley rats anesthetized by diethyl ether under aseptic conditions and washed in $\mathrm{Ca}^{2+}$ - and $\mathrm{Mg}^{2+}$-free phosphatebuffered saline (PBS). After the atria and aorta were discarded, the ventricles were minced and enzymatically digested with $0.1 \%$ collagenase type I (Sigma-Aldrich) and $0.125 \%$ trypsin (Gibco BRL Life Technologies, Inc.). The liberated cells were collected by centrifugation and incubated in $100-\mathrm{mm}$ culture dishes for $90 \mathrm{~min}$ at $37^{\circ} \mathrm{C}$ in a humidified incubator with $5 \% \mathrm{CO}_{2}$ air. Non-adherent cells were harvested as cardiomyocytes and seeded at a density of $1 \times 10^{6}$ cells/well into 6-well culture plates. They were incubated in DMEM supplemented with $10 \%$ FBS, $1 \%$ penicillin/streptomycin and bromodeoxyuridine (BrdU, $100 \mu \mathrm{M}$; Sigma-Aldrich). After $48 \mathrm{~h}$, the culture medium was replaced by DMEM containing $1 \%$ FBS. After $24 \mathrm{~h}$ of serum starvation, the cells were incubated with AA for $24 \mathrm{~h}$ prior to treatment with TGF- $\beta 1[4 \mathrm{ng} / \mathrm{ml}$, as previously described in the study by Lim et al (4)] stimulation, which lasted $24 \mathrm{~h}$. Untreated cells served as the controls. AA was freshly prepared as a stock solution in DMSO and diluted with sterile double-distilled water $[0.1 \%$ (v/v) DMSO]. TGF- $\beta 1$ was dissolved in sterile double-distilled water. There were 5 experimental groups: i) control, ii) AA, iii) TGF- $\beta 1$, iv) TGF- $\beta 1+$ vehicle (DMSOsaline) and v) TGF- $\beta 1+$ AA.

Cell viability assay. Cell viability was monitored using a CCK-8 assay according to the manufacturer's instructions. In brief, the cardiomyocytes were initially cultured at a density of $1 \times 10^{4}$ cells/well in 96-well plates. The cells were then pretreated with various concentrations of AA (2.5-30 $\mu \mathrm{M})$ for $24 \mathrm{~h}$. CCK-8 solution $(10 \mu \mathrm{l})$ was then added to each culture well followed by incubation for $4 \mathrm{~h}$ at $37^{\circ} \mathrm{C}$. The absorbance at $450 \mathrm{~nm}$ was measured using a microplate reader (Bio-Rad Laboratories, Hercules, CA, USA). All experiments were performed in triplicate, and cell viability was calculated as a percentage.

Immunofluorescence analysis of cardiomyocytes. The cardiomyocytes were cultured on coverslips. Following TGF- $\beta 1$ ( $4 \mathrm{ng} / \mathrm{ml}$ ) stimulation for $24 \mathrm{~h}$ in the presence or absence of AA for $24 \mathrm{~h}$, the cells were washed with PBS, fixed with $4 \%$ paraformaldehyde for $20 \mathrm{~min}$ and permeablized with $0.1 \%$ Triton X-100/PBS for $10 \mathrm{~min}$. After blocking with 5\% bovine serum for $30 \mathrm{~min}$, the size of the cells was determined by staining the membranes with specific anti-sarcomeric $\alpha$-actinin antibody (Sigma-Aldrich) and visualized under an inverted fluorescence microscope (Nikon, Tokyo, Japan). The size of the cardiomyocytes was determined using ImageJ software (NIH, Bethesda, MD, USA).

Animal models of cardiac hypertrophy. An animal model of pressure overload-induced cardiac hypertrophy was created by transverse aortic constriction (TAC) in male C57BL/6 mice (8-10 weeks of age, 20-30 g body weight; Experimental Animal Center of Jiangsu Province, Nanjing, China). To achieve constriction, a 7-0 suture was snugly tied twice around a blunt 27-gauge needle, which was positioned adjacent to the aorta between the right innominate and left carotid arteries and promptly removed following ligation (14). This produced a $60-70 \%$ constriction with an outer aortic diameter of approximately $0.4 \mathrm{~mm}$. Acute and chronic mortality from the ligature procedure was $<10 \%$. Sham-operated controls consisted of age-matched littermates 
that underwent an identical surgical procedure, including the isolation of the aorta, only without placement of the ligature. Twenty-four hours after the operation, the mice subjected to TAC and the sham-operated mice were orally gavaged with AA $100 \mathrm{mg} / \mathrm{kg} /$ day or the vehicle (DMSO-saline). After 2 weeks of $\mathrm{TAC}$, the hearts were harvested and the ratio of heart weight to body weight $(\mathrm{HW} / \mathrm{BW})$ was calculated. The heart samples were frozen in liquid nitrogen and stored at $-80^{\circ} \mathrm{C}$. AA was freshly prepared as a stock solution in DMSO and diluted with saline to yield a final AA concentration of $100 \mathrm{mg} / \mathrm{kg}$ body weight [0.1\% (v/v) DMSO] (based on our preliminary experiment). The vehicle control was administered a mixture of DMSO with saline $[0.1 \%$ (v/v) DMSO]. There were 5 experimental groups: i) sham-operated control (sham), ii) sham + AA, iii) untreated $\mathrm{TAC}$, iv) $\mathrm{TAC}+$ vehicle and v) $\mathrm{TAC}+\mathrm{AA}$.

Transthoracic echocardiography. All mice were anesthetized by a mixture of isoflurane $(1.5 \%)$ and oxygen $(0.5 \mathrm{l} / \mathrm{min})$. Cardiac dimensions and functions were evaluated by echocardiography (Vevo 2100 equipped with a 30-MHz high-resolution phase array transducer; VisualSonics, Toronto, ON, Canada) after 2 weeks following TAC. The left ventricle (LV) was assessed in both parasternal long-axis and short-axis views. End-systolic and end-diastolic volume was defined as the phase in which the smallest and largest area of the LV were obtained, respectively. Interventricular septal end-diastolic dimension (IVSD), left ventricular end diastolic posterior wall dimension (LVPWD), left ventricular end-systolic diameter (LVESD) and left ventricular end-diastolic diameter (LVEDD) were measured from the LV M-mode tracing with a sweep speed of $50 \mathrm{~mm} / \mathrm{sec}$ at the papillary muscle level. The percentage of fractional shortening $(\% \mathrm{FS})$ was calculated using a standard formula: $\% \mathrm{FS}=[(\mathrm{LVEDD}-\mathrm{LVESD}) / \mathrm{LVEDD}] \mathrm{x} 100$. At each location, for each mouse, 6-10 beats were analyzed.

Nuclear protein extraction and electrophoretic mobility shift assay (EMSA). Nuclear proteins were isolated from the LV samples and cultured cardiomyocytes, as described in a previous study (9). NF- $\mathrm{B}$ binding activity was examined using an EMSA kit (Pierce Biotechnology, Inc.), according to the manufacturer's instructions. For the competition assay, specific unlabeled NF- $\kappa \mathrm{B}$ competitors ( 200 -fold molar excess) were employed along with the binding reaction mixture. In brief, a biotin end-labeled DNA duplex of sequences containing the $\mathrm{NF}-\kappa \mathrm{B}$ binding domain (5'-AGTTGAGGGGACTTTCCC AGGC-3' and 3'-TCAACTCCCCTGAAAGGGTCCG-5') was incubated with nuclear proteins at room temperature for $20 \mathrm{~min}$. The reaction mixture was separated by $6.5 \%$ polyacrylamide gel electrophoresis, and transferred onto nylon membranes. The membranes were subjected to UV light cross-link for $1 \mathrm{~min}$ and were then incubated with blocking buffer containing stabilized streptavidin-horseradish peroxidase conjugate $(1: 2,000)$ for $15 \mathrm{~min}$. The signals on the membranes were detected with the Chemiluminescent Nucleic Acid Detection Module (Pierce Biotechnology, Inc.). The NF- $\kappa \mathrm{B}$ binding bands were scanned by G:BOX-CHEMI-XR5-E (Syngene, Frederick, MD, USA) and the relative intensities were analyzed using ImageJ software (NIH).

Quantitative RT-PCR (RT-qPCR). mRNA transcripts were quantified by RT-qPCR. Briefly, RNA from the LV tissues and cardiomyocytes was isolated using RNAiso and TRIzol reagent (Invitrogen, Carlsbad, CA, USA), respectively. cDNA generated from $500 \mathrm{ng}$ of total RNA was reverse transcribed using the PrimeScript $^{\text {TM }}$ RT reagent kit (Takara Biotechnology, Shiga, Japan). Specific products were determined using Eppendorf Mastercycler ep realplex analysis software according to the instructions provided with SYBR ${ }^{\circledR}$ Premix Ex Taq ${ }^{\mathrm{TM}}$ II (Tli RNaseH Plus; Takara Biotechnology). The specific forward and reverse primers used were as follows: mouse atrial natriuretic peptide (ANP) forward, 5'-CCAGCATGGGC TCCTTCTCCA-3' and reverse, 5'-CCGGAAGCTGTTGCA GCCTAGT-3'; mouse TGF- $\beta 1$ forward, 5 -GACTCTCCACCT GCAAGACC-3' and reverse, 5'-ACTGCTTCCCGAATGT CTGA-3'; mouse glyceraldehyde-3-phosphate dehydrogenase (GAPDH) forward, 5'-GGCATCGTGGAGGGA-3' and reverse, 5'-TGAGTTAGACTGAGTGAAGAG-3'; rat ANP forward, 5'-GCTCGAGCAGATCGCAAAAG-3' and reverse, 5'-CACC ACCTCTCAGTGGCAAT-3'; and rat GAPDH forward, 5'-ATGGGAAGCTGGTCATCAAC-3' and reverse, 5'-GTGG TTCACACCCATCACAA-3'. The expression levels of all transcripts were normalized to the housekeeping gene, GAPDH, in the same tissue. The relative mRNA expression was calculated

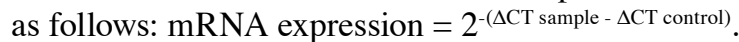

Western blot analysis. Total protein was extracted from the LV tissues and cardiac fibroblasts and assessed by western blot analysis and enhanced chemiluminescence. The proteins (20-30 $\mu \mathrm{g}$ ) were separated by 10-15\% SDS-PAGE and subsequenlty transferred onto polyvinylidene difluoride (PVDF) membranes using a Mini Trans-Blot electrophoresis transfer cell (Bio-Rad Laboratories). The membranes were incubated with appropriate primary antibodies against TGF- $\beta 1, \mathrm{p}-\mathrm{p} 38$ (Thr180/Tyr182), p38, p-ERK1/2 (Thr202/Tyr204), ERK1/2, p-JNK (Tyr183/Tyr185), JNK and GAPDH. After extensive washing in TBST $(10 \mathrm{mM}$ Tris- $\mathrm{HCl}, 150 \mathrm{mM} \mathrm{NaCl}$ and $0.1 \%$ Tween-20, $\mathrm{pH}$ 7.6), the membranes were incubated with the appropriate HRP-conjugated secondary antibody. The signals were detected using an ECL Western Blot Detection kit (Thermo Scientific, Rockford, IL, USA), and blot quantification was performed using densitometry with ImageJ software (NIH).

Histological analysis. The hearts were fixed in $10 \%$ neutral formalin, dehydrated in 75,80,90 and 100\% ethanol,transferred to xylene, embedded in paraffin and sectioned at a thickness of 4-5 $\mu \mathrm{m}$, then stained with hematoxylin and eosin (H\&E) or with Masson's trichrome stain. The diameter of the cardiomyocytes and the interstitial collagen fraction were measured using NIH ImageJ software (NIH). At least 3 different hearts with 5 separate fields of cells (total 50-70 cells for each heart), were quantified for cellular analysis.

Statistical analysis. Data are expressed as the means \pm standard deviation (SD). The GraphPad Prism 5.01 (GraphPad Software, Inc., La Jolla, CA, USA) and PASW Statistics 18.0 (SPSS Inc., Fayetteville, NC, USA) packages were used. Differences among groups were tested by one-way ANOVA. Comparisons between 2 groups were performed by an unpaired two-tailed Student's t-test. If a p-value was $<0.05$, the result was considered statistically significant. 
A

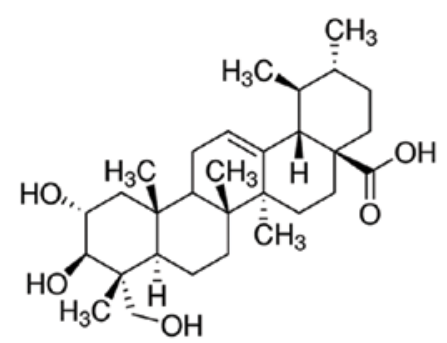

C

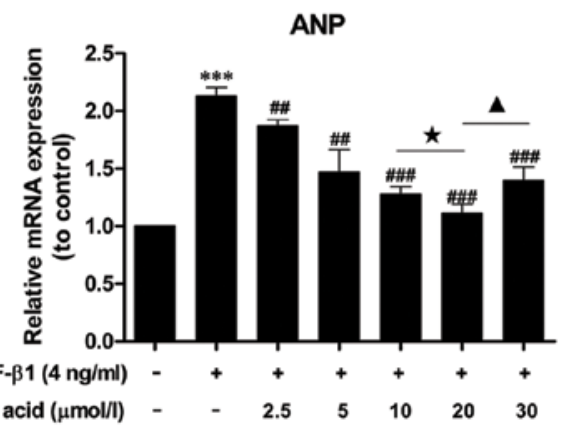

B
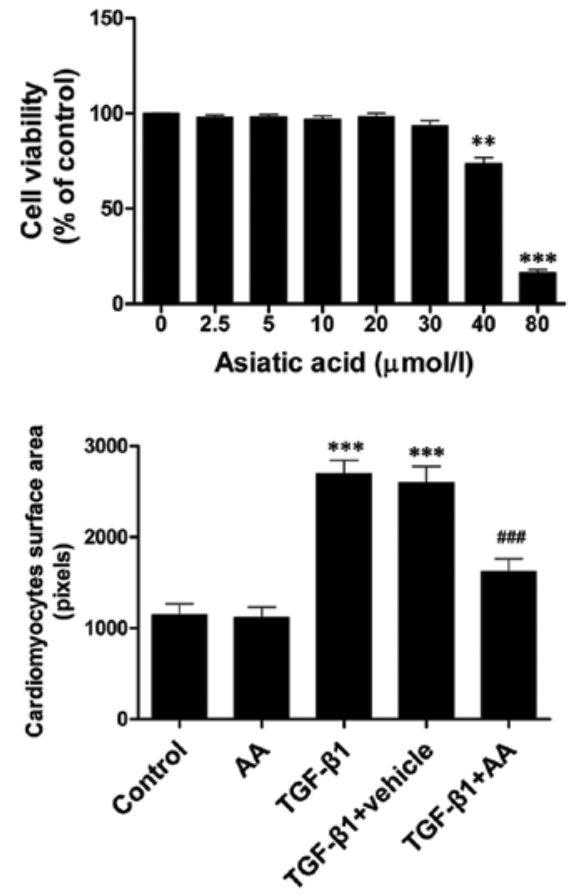

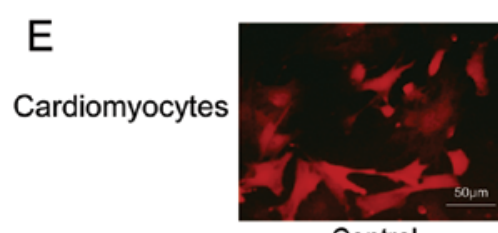

Control

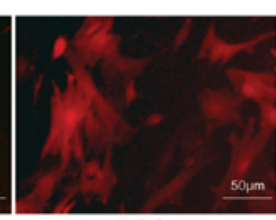

AA

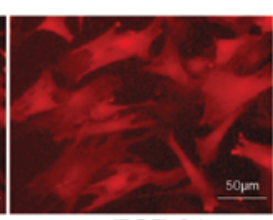

TGF- $\beta 1$

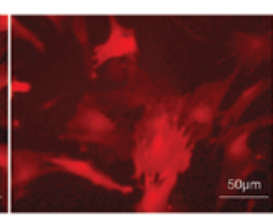

TGF- $\beta 1+$ vehicle

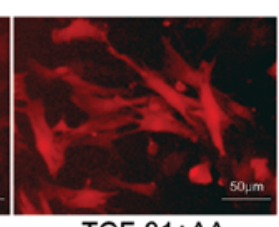

TGF- $\beta 1+A A$

Figure 1. Protective effects of asiatic acid (AA) on transforming growth factor- $\beta 1$ (TGF- $\beta 1$ )-induced hypertrophic response in cardiomyocytes. (A) The chemical structure of AA. (B) Viability of cardiomyocytes was examined by CCK-8 assay. (C) Cardiomyocytes were pre-treated with various concentrations of AA for $24 \mathrm{~h}$, then stimulated with TGF- $\beta 1$ ( $4 \mathrm{ng} / \mathrm{ml})$ for $24 \mathrm{~h}$ and the mRNA expression of atrial natriuretic peptide (ANP) was measured. (D and E) The cells were treated with AA $(20 \mu \mathrm{M})$ for $24 \mathrm{~h}$ prior to TGF- $\beta 1(4 \mathrm{ng} / \mathrm{ml})$ stimulation for $24 \mathrm{~h}$. Neonatal cardiomyocytes were stained with anti- $\alpha$-actinin and analyzed by immunofluorescence. Images of representative cardiomyocytes of each group are shown in (E) and the surface areas of cardiomyocytes are shown in $(\mathrm{D})$; $\mathrm{n}=3$. ${ }^{* *} \mathrm{P}<0.01$ and ${ }^{* * * *} \mathrm{P}<0.001$ vs. untreated cells; ${ }^{\# \#} \mathrm{P}<0.01$ and ${ }^{\# \# \#} \mathrm{P}<0.001$ vs. TGF- $\beta 1$-treated cells; ${ }^{\star} \mathrm{P}<0.05$ vs. treatment with $10 \mu \mathrm{M}$ AA; ${ }^{\wedge} \mathrm{P}<0.05$ vs. treatment with $30 \mu \mathrm{M} \mathrm{AA}$.

\section{Results}

AA inhibits the TGF- $\beta 1$-induced hypertrophic response in cardiomyocytes. TGF- $\beta 1$ stimulation has been demonstrated to induce hypertrophic effects on cardiomyocytes, promoting the synthesis of fetal contractile proteins (5). To determine whether AA inhibits cardiomyocyte hypertrophy induced by TGF- $\beta 1$, the cells were treated with TGF- $\beta 1(4 \mathrm{ng} / \mathrm{ml})$ for $24 \mathrm{~h}$ in the presence or absence of AA $(2.5-30 \mu \mathrm{M})$. The expression of the fetal gene, ANP, was examined by RT-qPCR. TGF- $\beta 1$ ( $4 \mathrm{ng} / \mathrm{ml})$ stimulation markedly increased the mRNA expression of ANP by 2.13 -fold relative to the control group $(\mathrm{P}<0.001)$ (Fig. 1C). AA $(2.5-30 \mu \mathrm{M})$ pre-treatment significantly decreased ANP mRNA expression, and the level of ANP mRNA expression in the AA-treated $(20 \mu \mathrm{M})$ group was $47.89 \%$ lower than that in the TGF- $\beta 1$-stimulated group $(\mathrm{P}<0.001)$.

In addition, the size of the cardiomyocytes was measured by immunofluorescence staining. TGF- $\beta 1$ stimulation induced a noticeable hypertrophic response in the cardiomyocytes that was not observed in the untreated control cells (Fig. 1D and E). By contrast, AA $(20 \mu \mathrm{M})$ pre-treatment attenuated the TGF- $\beta 1$ induced hypertrophic response. However, the AA-treated cells were the same size as the untreated cells.
AA prevents the TGF- $\beta 1$-stimulated increase in 38 and ERK1/2 phosphorylation and $N F-\kappa B$ binding activity. The effects of AA on TGF- $\beta 1$-stimulated NF- $\kappa$ B binding activity and MAPK phosphorylation were examined. The phosphorylation levels of p38 and ERK1/2, as well as the NF- $\kappa \mathrm{B}$ binding activity were markedly higher after $24 \mathrm{~h}$ of TGF- $\beta 1(4 \mathrm{ng} / \mathrm{ml})$ stimulation compared with the untreated cells $(\mathrm{P}<0.001, \mathrm{P}<0.001$ and $\mathrm{P}<0.01$ for $\mathrm{p} 38, \mathrm{ERK} 1 / 2$ and $\mathrm{NF}-\kappa \mathrm{B}$, respectively) (Fig. 2). However, TGF- $\beta 1$ did not induce the phosphorylation of JNK. AA pre-treatment substantially reduced the TGF- $\beta 1$-stimulated increase in the levels of p-p38/p38 and p-ERK1/2/ERK1/2 and $\mathrm{NF}-\kappa \mathrm{B}$ binding activity $(\mathrm{P}<0.001, \mathrm{P}<0.001$ and $\mathrm{P}<0.01$ for $\mathrm{p} 38$, ERK1/2 and NF- $\kappa \mathrm{B}$, respectively).

AA administration attenuates TAC-induced cardiac hypertrophy and cardiac dysfunction. The issue of whether AA can attenuate pressure overload-induced cardiac hypertrophy was then addressed. The mice were subjected to TAC and treated with AA (100 mg/kg/daily) or with the vehicle for 2 weeks. Pressure overload induced by TAC markedly increased the ratio of $\mathrm{HW} / \mathrm{BW}$ to $42.06 \%$ higher than that in the sham group $(\mathrm{P}<0.001)$ (Fig. 3A). AA administration reduced the ratio of $\mathrm{HW} / \mathrm{BW}$ by $28.27 \%$ relative to the TAC group 


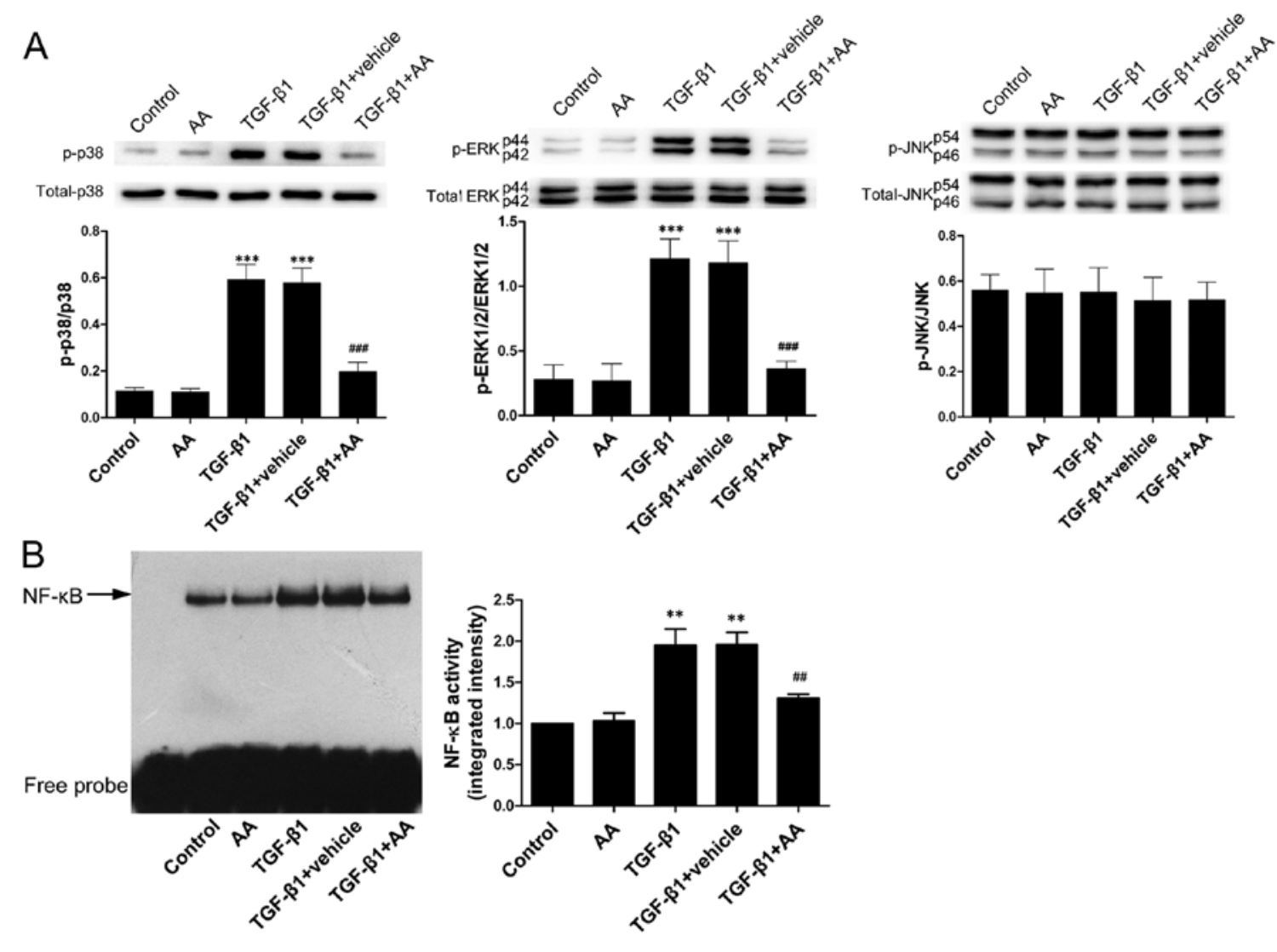

Figure 2. Asiatic acid (AA) prevents the transforming growth factor- $\beta 1$ (TGF- $\beta 1$ )-induced p38 and extracellular signal-regulated kinase (ERK)1/2 phosphorylation and nuclear factor- $\mathrm{kB}$ (NF- $\mathrm{kB}$ ) activation in cardiomyocytes. (A) The levels of phosphorylated (p)-p38/p38, p-ERK1/2/ERK1/2 and p-c-Jun N-terminal kinase (JNK)/JNK were determined by western blot analysis. (B) NF-kB binding activity was determined by electrophoretic mobility shift assay (EMSA); n=3. ${ }^{* * *} \mathrm{P}<0.01$ and ${ }^{* * * *} \mathrm{P}<0.001$ vs. untreated cells; ${ }^{\# \#} \mathrm{P}<0.01$ and ${ }^{\# \# "} \mathrm{P}<0.001$ vs. TGF- $\beta 1$-treated cells.

$(\mathrm{P}<0.001)$. The mice subjected to pressure overload displayed markedly higher IVSD and LVPWD compared with the the sham-operated mice $(\mathrm{P}<0.001$ and $\mathrm{P}<0.001$ for IVSD and LVPWD, respectively) (Fig. 3B and C). AA administration reduced the TAC-induced increase in IVSD and LVPWD by 22.24\% $(\mathrm{P}<0.001)$ and 33.28\% $(\mathrm{P}<0.001)$, respectively. LVEDD decreased after 2 weeks of pressure overload $(\mathrm{P}<0.05)$, and AA administration prevented the TAC-induced decrease in LVEDD $(\mathrm{P}<0.05)$. In addition, \%FS significantly decreased by $22.25 \%$ relative to the sham group at 2 weeks after TAC $(\mathrm{P}<0.001)$. AA pre-treatment attenuated the TAC-induced decrease in $\% \mathrm{FS}(\mathrm{P}<0.001)$.

Pressure overload-induced cardiac hypertrophy was evidenced by H\&E staining; the cross-sectional area (CSA) of the cardiomyocytes in the TAC group was much larger than that of the cells in the sham group $(\mathrm{P}<0.001)$ (Fig. 4A and $\mathrm{B})$. AA reduced the TAC-induced increase in CSA $(\mathrm{P}<0.01)$. ANP mRNA expression is considered as a predominant hallmark of hypertrophic remodeling (15). Fig. 4C illustrates that pressure overload significantly increased the mRNA expression of ANP in the myocardium by 1.92-fold compared to that of the sham group $(\mathrm{P}<0.001)$. AA prevented the $\mathrm{TAC}$-induced increase in ANP mRNA expression $(\mathrm{P}<0.001)$. In addition, the expression of TGF- $\beta 1$ mRNA and protein in the pressure-overloaded myocardium was markedly higher than in the sham group (Fig. 4D and $\mathrm{E})(\mathrm{P}<0.001$ and $\mathrm{P}<0.001$ for TGF- $\beta 1 \mathrm{mRNA}$ and protein, respectively). By contrast, AA administration substantially reduced the TAC-induced increase in the expression of TGF- $\beta 1$ mRNA and protein (Fig. 4D and E) $(\mathrm{P}<0.001$ and $\mathrm{P}<0.01$ for TGF- $\beta 1$ mRNA and protein, respectively).

AA prevents the TAC-induced increase in p38 and ERK1/2 phosphorylation and $N F-\kappa B$ binding activity. The activation of MAPKs and NF- $\kappa \mathrm{B}$ has been shown to play an important role in the development of cardiac hypertrophy induced by TAC $(3,9,16)$. We thus examined the effect of AA on TAC-induced MAPK phosphorylation and NF- $\kappa \mathrm{B}$ binding activity. The phosphorylation levels of $\mathrm{p} 38$ and ERK1/2 and the NF- $\kappa \mathrm{B}$ binding activity were notably higher in the TAC group compared with the sham group following pressure overload lasting for 2 weeks (Fig. 4F and $\mathrm{G})$. However, TAC did not induce the activation of JNK. By contrast, AA administration prevented the TAC-induced increase in the levels of p-p38/p38 and p-ERK1/2/ERK1/2 and in the NF- $\kappa \mathrm{B}$ binding activity $(\mathrm{P}<0.05, \mathrm{P}<0.01$ and $\mathrm{P}<0.001$ for p38, ERK1/2 and NF- $\mathrm{B}$, respectively).

\section{Discussion}

The present study is among the first to attempt to elucidate the biological effects of AA on cardiac hypertrophy. Our results indicated that AA prevented not only the cardiomyocyte hypertrophic response induced by TGF- $\beta 1$ in vitro, but also 
A

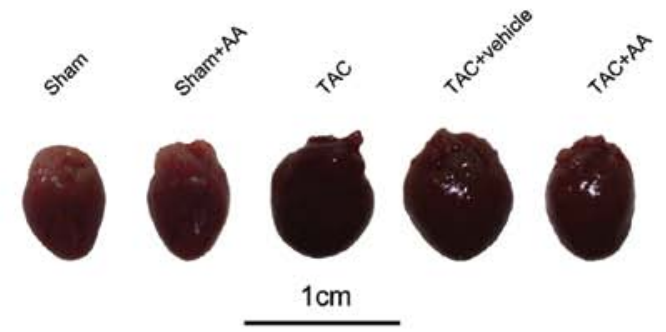

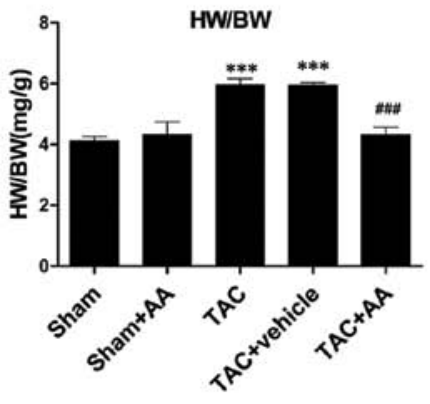

B
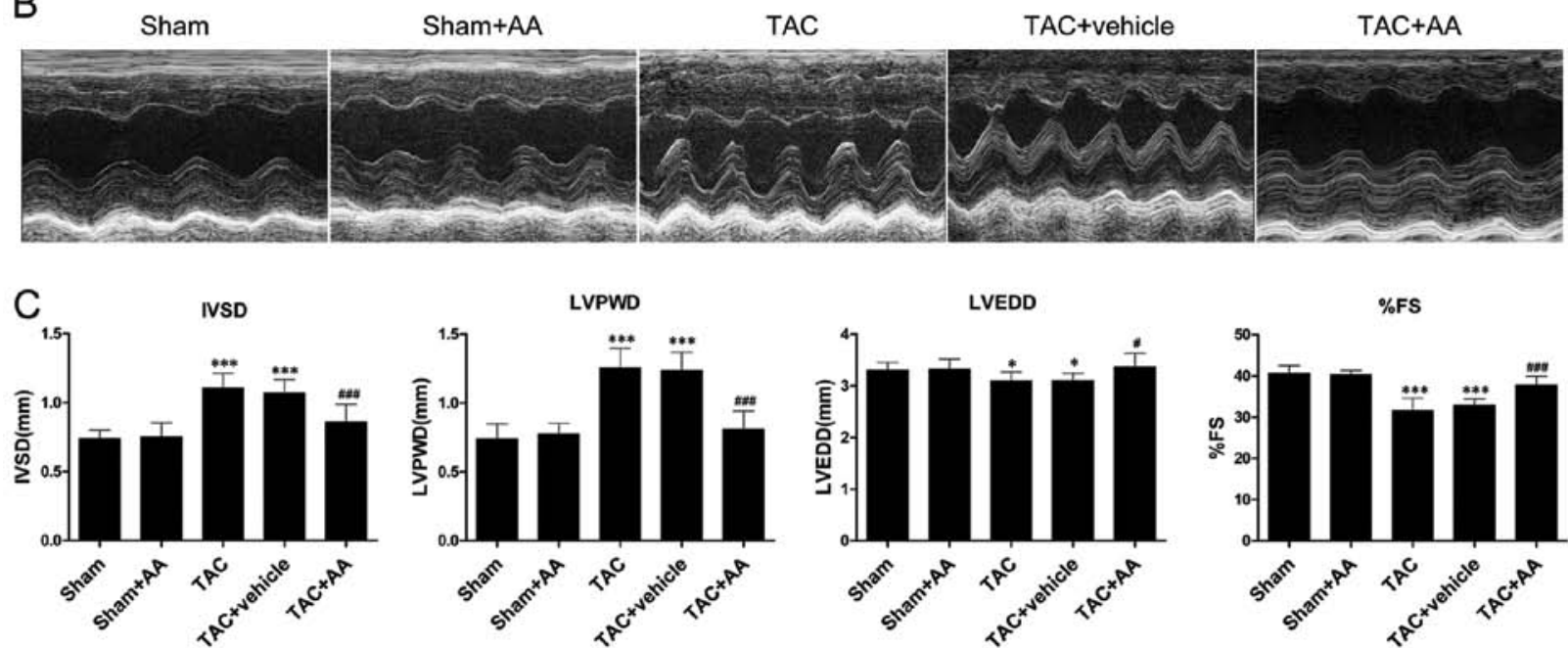

Figure 3. Asiatic acid (AA) administration prevents transverse aortic constriction (TAC)-induced cardiac dysfunction. (A) Representative images of hearts showing TAC-induced cardiac hypertrophy and the heart weight to body weight (HW/BW) ratio. (B) Representative transthoracic M-mode echocardiogram for each group. (C) Bar graphs represent the mean \pm standard deviation (SD) of IVSD, LVPWD, LVEDD and percentage fractional shortening (\%FS). $\mathrm{n}=10$ mice/group, ${ }^{*} \mathrm{P}<0.05$ and ${ }^{* * *} \mathrm{P}<0.001$ vs. sham (control) group; ${ }^{\#} \mathrm{P}<0.05$ and ${ }^{\# \# \#} \mathrm{P}<0.001$ vs. TAC group.

the upregulation of TGF- $\beta 1$ levels and cardiac hypertrophy induced by TAC in vivo. The anti-hypertrophic effects of AA were found to be associated with the reduction in TGF- $\beta 1$ expression, the deactivation of p38 and ERK1/2 and the inhibition of $\mathrm{NF}-\kappa \mathrm{B}$ binding activity. These findings support the conclusion that AA is a suitable candidate for the prevention and treatment of cardiac hypertrophy.

TGF- $\beta 1$ is a pleiotropic and multifunctional cytokine, which serves as a master switch in the pathogenesis of cardiac hypertrophy $(4,5)$. It has been previously demonstrated that TGF- $\beta 1$ levels substantially increase in the pressure-overloaded myocardium during hypertrophic growth (17). Moreover, the role of TGF- $\beta 1$ in provoking cardiac hypertrophy is evidently supported in transgenic mice. The overexpression of TGF- $\beta 1$ in transgenic mice has been shown to contribute significantly to cardiac hypertrophy, with other factors being contractile dysfunction and interstitial fibrosis (18). Conversely, TGF- $\beta$ deficient mice show no marked cardiac hypertrophy in response to hypertrophic stimuli (4). In accordance with these reports, the in vitro experiments of the present study revealed that TGF- $\beta 1$ stimulation markedly increased the size of the cardiomyocytes, as well as the mRNA expression of ANP, which are characteristics of hypertrophic processes (15). In the present in vivo pressure overload model, the mice subjected to TAC displayed a marked upregulation in TGF- $\beta 1$ mRNA and protein levels in the myocardium, as well as significant cardiac hypertrophy. AA treatment was found to prevent the
TGF- $\beta 1$-induced hypertrophic response of the cardiomyocytes. In vivo experiments revealed that AA treatment not only reduced the expression of TGF- $\beta 1$ mRNA and protein in the pressure-overloaded myocardium, but also attenuated cardiac hypertrophy and improved cardiac performance by reducing the dimensions of the left ventricular chamber. This further demonstrates that TGF- $\beta 1$ serves as a trigger for cardiac hypertrophy induced by pressure overload. Taken together, these data suggest that the inhibition of TGF- $\beta 1$ signaling may be one of the mechanisms through which AA attenuates cardiac hypertrophy.

The activation of MAPKs, such as p38, ERK1/2 and JNK, has been implicated in cardiac hypertrophy $(3,19)$. p38 and JNK have been reported to significantly contribute to the induction of specific gene expression and increased protein synthesis in the hypertrophic myocardium $(20,21)$. ERK1/2 has been implicated in growth-associated hypertrophic growth (22). More importantly, extensive basic and clinical studies have demonstrated that MAPKs serve as downstream signaling targets of TGF- $\beta 1(3,5,6)$. For instance, Huang et al $(7)$ reported that TGF- $\beta 1$ signaling induced the phosphorylation of p38, ERK1/2 and JNK, and was largely responsible for cardiac hypertrophy in the pressure-overloaded myocardium in vivo. In in vitro experiments, Lim et al (4) observed that the phosphorylation levels of p38, ERK1/2 and JNK significantly increased in cardiomyocytes following TGF- $\beta 1$-induced hypertrophic growth. In addition, Esposito et al (23) reported that the phosphorylation 
A

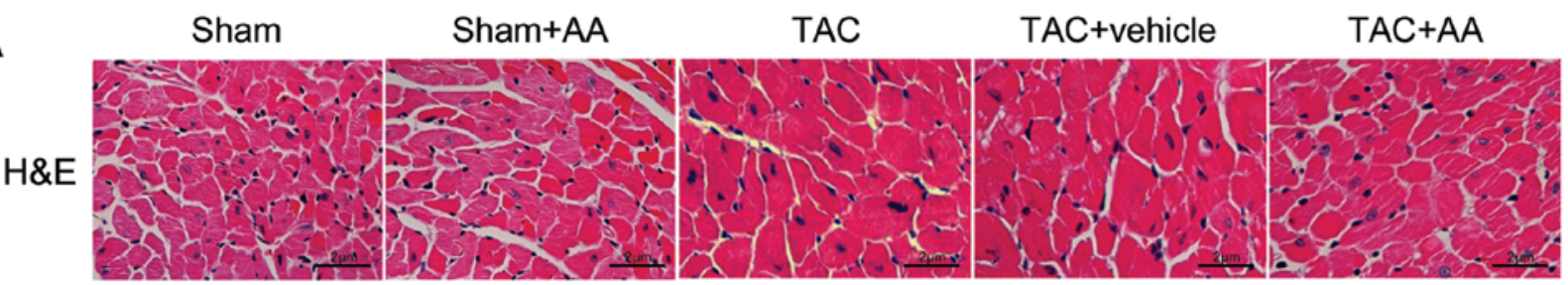

B
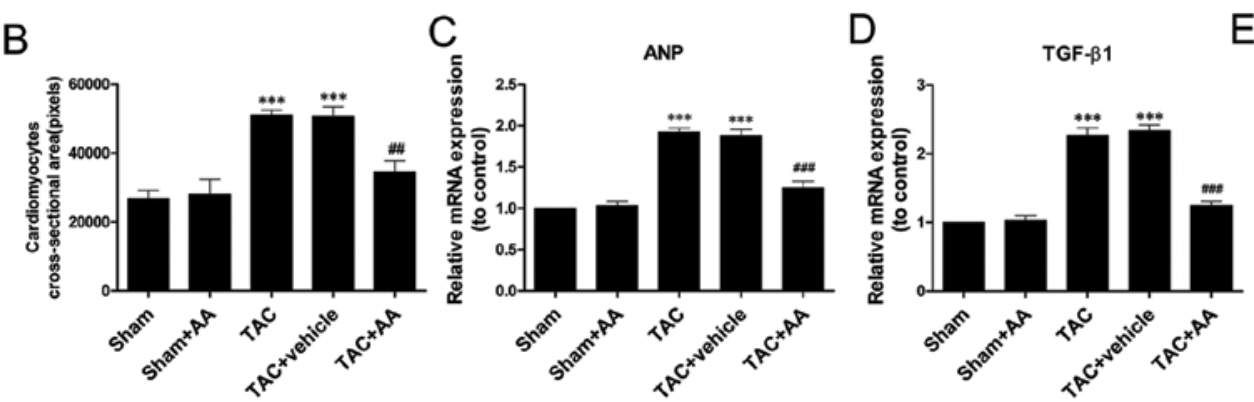

$\mathrm{E}_{\mathrm{TGF}-\beta 1}=-\mathbf{- a c t i n}=$

$\mathrm{F}$
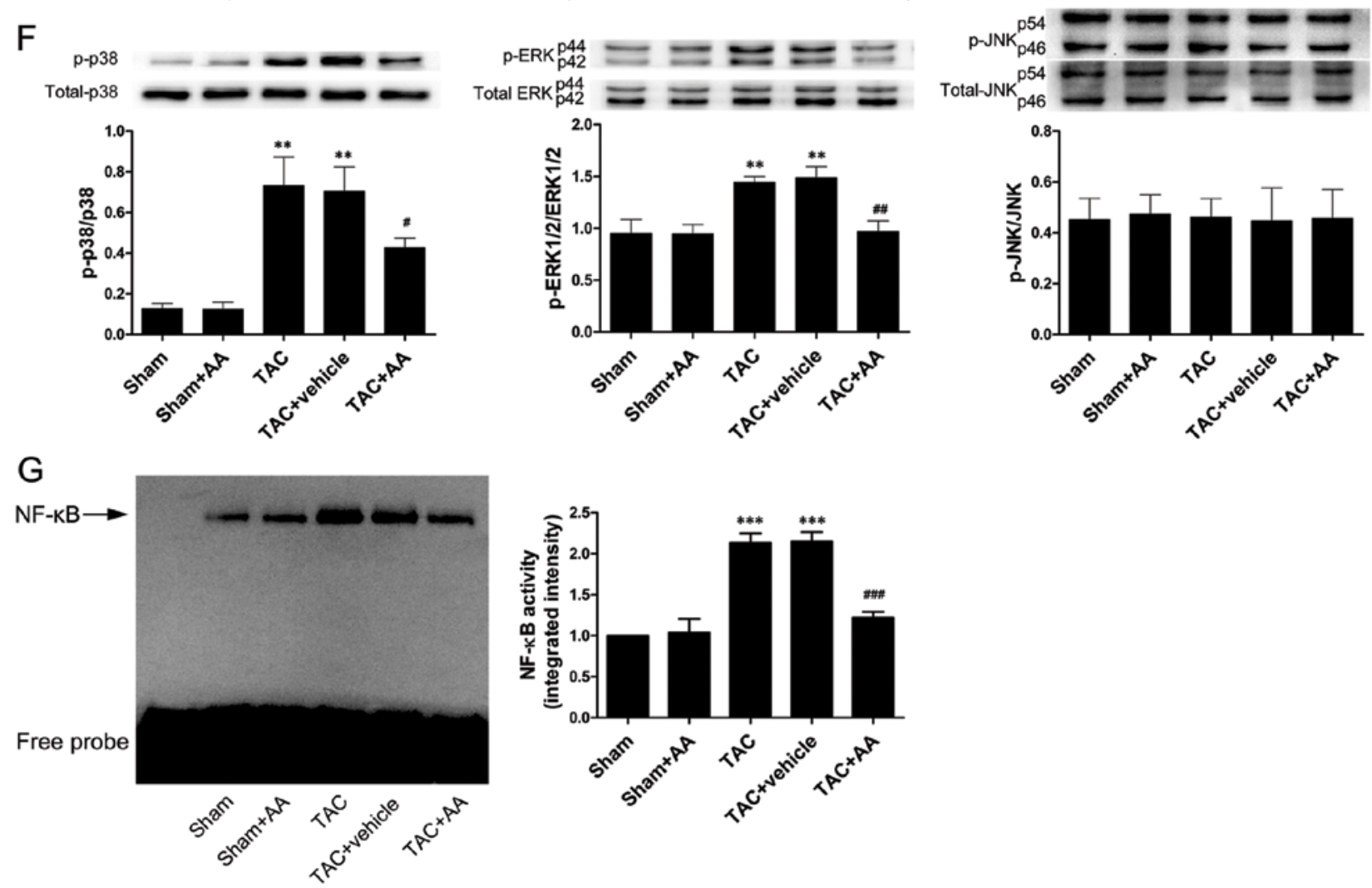

Figure 4. Asiatic acid (AA) attenuates transverse aortic constriction (TAC)-induced cardiac hypertrophy, p38 and extracellular signal-regulated kinase (ERK)1/2 phosphorylation, and NF- $\mathrm{BB}$ activation in the myocardium. (A) Heart sections were counterstained with hematoxylin and eosin (H\&E). Representative images demonstrating cardiac hypertyophy of heart sections from each group; magnification, $\mathrm{x} 400$ ( $\mathrm{n}=3$ mice/group). (B) Cardiomyocyte cross-sectional area. (C) Levels of atrial natriuretic peptide (ANP) mRNA expression. (D) Levels of transforming growth factor- $\beta 1$ (TGF- $\beta 1$ ) mRNA expression. (E) Levels of TGF- $\beta 1$ protein expression. (F) Levels of phosphorylated (p)-p38/p38, p-ERK1/2/ERK1/2 and p-c-Jun N-terminal kinase (JNK)/JNK were determined by western blot analysis. (G) NF- $\kappa$ B binding activity was determined by electrophoretic mobility shift assay (EMSA). $\mathrm{n}=10$ mice/group, ${ }^{* *} \mathrm{P}<0.01$ and ${ }^{* * *} \mathrm{P}<0.001 \mathrm{vs}$. sham (control) group; ${ }^{\#} \mathrm{P}<0.05,{ }^{\#} \mathrm{P}<0.01$ and ${ }^{\# \# \#} \mathrm{P}<0.001$ vs. TAC group.

levels of p38 and ERK1/2 significantly increased following treatment with TAC for 1 week. JNK activity also increased, but decreased over time. In accordance with these studies, our results showed a significant increase in the phosphorylation levels of p38 and ERK1/2 in cardiomyocytes following TGF- $\beta 1$-induced hypertrophy in vitro, but no increase was observed for JNK. The present in vivo experiments showed that the significant increase in the p38 and ERK1/2 phosphorylation levels positively correlated with the marked upregulation of TGF- $\beta 1$ expression in the pressure-overloaded myocardium. This indicated that, in the present study, $\mathrm{p} 38$ and ERK1/2 served as downstream signaling targets of TGF- $\beta 1$. When AA was administered, the TGF- $\beta 1$ induced increase in the p38 and ERK1/2 phosphorylation levels markedly reduced in vitro and in vivo.

$\mathrm{NF}-\kappa \mathrm{B}$, a DNA-binding transcription factor, is known to play a critical role in controlling the production of proinflammatory cytokines and is required for the development of cardiac hypertrophy $(8,16,24,25)$. As previously described, 
MAPK signaling can phosphorylate and activate their target proteins and transcription factors, resulting in NF- $\mathrm{\kappa B}$ translocation to the nucleus. This initiates the expression of fetal genes and the production of inflammatory cytokines, and ultimately provokes the development of cardiac hypertrophy $(6,8,24,26)$. On the other hand, the inhibition of NF- $\kappa$ B binding activity may be a means of preventing cardiac hypertrophy $(8,26)$. For instance, the inactivation of NF- $\mathrm{KB}$ with direct gene transfection of sh-p65 RNA has been shown to result in the attenuation of cardiac hypertrophy (26). In addition, Li et al (8) reported that blocking NF- $\mathrm{KB}$ binding activity in the myocardium significantly attenuated the pressure overload-induced cardiac hypertrophy. In accordance with these findings, the present study found that the NF- $\mathrm{kB}$ binding activity was significantly increased in cardiomyocytes following TGF- $\beta 1$-induced hypertrophy in vitro. In vivo experiments revealed that the significant increase in NF- $\kappa \mathrm{B}$ binding activity was associated with the marked activation of TGF- $\beta 1-p 38 /$ ERK1/2 signaling in the pressure-overloaded myocardium. AA administration substantially reduced the NF- $\mathrm{kB}$ binding activity in the TGF- $\beta 1$-stimulated hypertrophic cardiomyocytes in vitro and in the pressure-overloaded myocardium in vivo.

In conclusion, to the best of our knowledge, the present study is the first to demonstrate that AA inhibits the development of cardiac hypertrophy in vitro and in vivo. The mechanisms through which AA attenuates this hypertrophic process involve the downregulation of TGF- $\beta 1$ expression levels, the inhibition of $\mathrm{p} 38$ and ERK1/2 phosphorylation, and the reduction in NF- $\mathrm{KB}$ binding activity. These results suggest that AA may be used as a pharmacological agent for the prevention and treatment of cardiac hypertrophy.

\section{Acknowledgements}

This study was supported in part by grants from the Natural Science Foundation of Jiangsu Higher Education Institutions (12KJB320003); the Administration of Traditional Chinese Medicine of Jiangsu Province (lz13217); the Nanjing Foundation for Development of Science and Technology (201303036); the National Natural Science Foundation of China (81300128); $\mathrm{Ph} . D$. Programs Foundation of Ministry of Education of China (20123234120015); the Jiangsu Natural Science Foundation (BK20131025); the Project Sponsored by the Scientific Research Foundation for Returned Overseas Chinese Scholars, State Education Ministry; and the Project Funded by the Priority Academic Program Development of Jiangsu Higher Education Institutions.

\section{References}

1. Frohlich ED and Susic D: Pressure overload. Heart Fail Clin 8 21-32, 2012.

2. Ruwhof $C$ and van der Laarse A: Mechanical stress-induced cardiac hypertrophy: mechanisms and signal transduction pathways. Cardiovasc Res 47: 23-37, 2000.

3. Heineke J and Molkentin JD: Regulation of cardiac hypertrophy by intracellular signalling pathways. Nat Rev Mol Cell Biol 7 : 589-600, 2006.

4. Lim JY, Park SJ, Hwang HY, Park EJ, Nam JH, Kim J and Park SI: TGF-beta1 induces cardiac hypertrophic responses via PKC-dependent ATF-2 activation. J Mol Cell Cardiol 39: 627-636, 2005
5. Dobaczewski M, Chen W and Frangogiannis NG: Transforming growth factor (TGF) $-\beta$ signaling in cardiac remodeling. J Mol Cell Cardiol 51: 600-606, 2011.

6. Derynck $R$ and Zhang YE: Smad-dependent and Smad-independent pathways in TGF-beta family signalling. Nature 425: 577-584, 2003.

7. Huang H, Tang QZ, Wang AB, et al: Tumor suppressor A20 protects against cardiac hypertrophy and fibrosis by blocking transforming growth factor-beta-activated kinase 1-dependent signaling. Hypertension 56: 232-239, 2010.

8. Li Y,Ha T, Gao X, et al: NF-kappaB activation is required for the development of cardiac hypertrophy in vivo. Am J Physiol Heart Circ Physiol 287: H1712-H1720, 2004.

9. Li HL, Wang AB, Huang Y, et al: Isorhapontigenin, a new resveratrol analog, attenuates cardiac hypertrophy via blocking signaling transduction pathways. Free Radic Biol Med 38: 243-257, 2005.

10. Pittella F, Dutra RC, Junior DD, Lopes MT and Barbosa NR: Antioxidant and cytotoxic activities of Centella asiatica (L) Urb. Int J Mol Sci 10: 3713-3721, 2009.

11. Yun KJ, Kim JY, Kim JB, et al: Inhibition of LPS-induced NO and PGE2 production by asiatic acid via NF-kappa B inactivation in RAW 264.7 macrophages: possible involvement of the IKK and MAPK pathways. Int Immunopharmacol 8: 431-441, 2008.

12. Zhang X, Wu J, Dou Y, Xia B, Rong W, Rimbach G and Lou Y: Asiatic acid protects primary neurons against C2-ceramide-induced apoptosis. Eur J Pharmacol 679: 51-59, 2012.

13. Tang LX, He RH, Yang G, et al: Asiatic acid inhibits liver fibrosis by blocking TGF-beta/Smad signaling in vivo and in vitro. PLoS One 7: e31350, 2012

14. Xu XH, Xu J, Xue L, Cao HL, Liu X and Chen YJ: VEGF attenuates development from cardiac hypertrophy to heart failure after aortic stenosis through mitochondrial mediated apoptosis and cardiomyocyte proliferation. J Cardiothorac Surg 6: 54, 2011.

15. Feng JA, Perry G, Mori T, Hayashi T, Oparil S and Chen YF: Pressure-independent enhancement of cardiac hypertrophy in atrial natriuretic peptide-deficient mice. Clin Exp Pharmacol Physiol 30: 343-349, 2003.

16. Zhu Y, Li T, Song J, et al: The TIR/BB-loop mimetic AS-1 prevents cardiac hypertrophy by inhibiting IL-1R-mediated MyD88-dependent signaling. Basic Res Cardiol 106: 787-799, 2011.

17. Li JM and Brooks G: Differential protein expression and subcellular distribution of TGFbeta1, beta2 and beta3 in cardiomyocytes during pressure overload-induced hypertrophy. J Mol Cell Cardiol 29: 2213-2224, 1997.

18. Rosenkranz S, Flesch M, Amann K, et al: Alterations of beta-adrenergic signaling and cardiac hypertrophy in transgenic mice overexpressing TGF-beta(1). Am J Physiol Heart Circ Physiol 283: H1253-H1262, 2002.

19. Wang Y: Mitogen-activated protein kinases in heart development and diseases. Circulation 116: 1413-1423, 2007.

20. LaMorte VJ, Thorburn J, Absher D, et al: Gq- and ras-dependent pathways mediate hypertrophy of neonatal rat ventricular myocytes following alpha 1-adrenergic stimulation. J Biol Chem 269: 13490-13496, 1994.

21. Wang Y: Signal transduction in cardiac hypertrophy-dissecting compensatory versus pathological pathways utilizing a transgenic approach. Curr Opin Pharmacol 1: 134-140, 2001.

22. Clerk A and Sugden PH: Untangling the Web: specific signaling from PKC isoforms to MAPK cascades. Circ Res 89: 847-849, 2001.

23. Esposito G, Prasad SV, Rapacciuolo A, Mao L, Koch WJ and Rockman HA: Cardiac overexpression of a G(q) inhibitor blocks induction of extracellular signal-regulated kinase and c-Jun $\mathrm{NH}(2)$-terminal kinase activity in in vivo pressure overload. Circulation 103: 1453-1458, 2001.

24. Hall G, Hasday JD and Rogers TB: Regulating the regulator: NF-kappaB signaling in heart. J Mol Cell Cardiol 41: 580-591, 2006.

25. Gordon JW, Shaw JA and Kirshenbaum LA: Multiple Facets

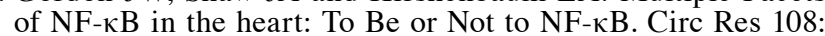
1122-1132, 2011.

26. Gupta S, Young D, Maitra RK, et al: Prevention of cardiac hypertrophy and heart failure by silencing of NF-kappaB. J Mol Biol 375: 637-649, 2008. 